\title{
Propranolol is Highly Effective to Treat Refeeding Oedema in a Girl with Anorexia Nervosa - A Case Report
}

Buchhorn R*, Bauman C and Willaschek C

Caritas Krankenhaus Bad Mergentheim, Department of

Pediatrics, Germany

*Corresponding author: Reiner Buchhorn, Caritas

Krankenhaus Bad Mergentheim, Department of

Pediatrics, Uhlandstr 7, 97980 Bad Mergentheim,

Germany

Received: February 14, 2017; Accepted: February 20, 2017; Published: February 22, 2017

\section{Editorial}

Edemas occur as a result of reinstitution of nutrition to patients who are severely malnourished due to anorexia nervosa, starvation or severe illness [1]. This refeeding oedema seems to be a part of the so called refeeding syndrome with a significant mortality in highly developed medical systems but most of all in starved children in the developing world [2]. The metabolic disturbances of the refeeding syndrome are well understood and investigated. However cardiac involvement is highly speculative although mortality is related to oedema and sudden cardiac death [3]. Within the last ten years we developed a pathophysiological model based on 24 hours heart rate variability analysis to explain the impact of nutrition on the autonomic nervous system [4]. These data may explain bradycardia in children with anorexia nervosa and the autonomic shift to sympathetic activation during refeeding.

In 1995 we had developed a pathophysiological model to explain the therapeutic effect of propranolol on oedema in children with single ventricle after the Fontan's procedure. The mechanism involved seems to be an increased mean capillary filtration pressure, calculated from hemodynamic data based on Starling's theories of ultra filtration in the capillaries [5].

Based upon these two pathophysiological models we recently treat a 14 years old girl with anorexia nervosa who developed leg oedema during refeeding with $1 \mathrm{mg} / \mathrm{kg}$ propranolol: Anorexia nervosa in this girl was a "complication" of in hospital treatment of obesity with weight reduction of $35 \mathrm{~kg}$. During refeeding the girl developed leg oedema that spontaneously resolved in our department using the NICE protocol for refeeding (http://www.eastcheshire. nhs.uk/About-TheTrust/policies/N/Nutrition\%20\%20Refeeding\%20 Syndrome\%20Guidelines\%20ECT2366.pdf)

However after slowly augmentation of nutrition up to $3500 \mathrm{kcal}$ using 1) high energy liquid nutrition and substitution 2) Vitamin B, Zinc, Potassium, Calcium Glycero phosphate, magnesium and omega-3-fatty acid supplementation, the girl developed severe leg oedema again. We observe no metabolic derangement like hypophosphatemia, hypokalemia and hypomagnesemia but dilution effects like decrease of hematocrit and plasma protein (Table 1). Despite normal ventricular function echocardiography showed little pericardial effusion and the NT-Pro-BNP values increase up to $869 \mathrm{pg} /$ $\mathrm{ml}$ indicating elevated atrial filling pressures. In this situation we decide to treat with $0.5 \mathrm{mg} / \mathrm{kg}$ propranolol uptitrated to $1 \mathrm{mg} / \mathrm{kg}$ after two days on our intensive care ward. We use the device NutriPlus $\odot$ (Data Input, Pöcking, Germany) for Bio Impedance Analysis (BIA).

Daily bodyweight monitoring shows the effect of the NICE refeeding protocol in the first treatment week and the immediate effect of propranolol in the fourth treatment week on oedema (Figure 1). Beside the laboratory parameters the table shows the results of the BIA and Holter ECG analysis. The equilibrium bodyweight seems to be $44 \mathrm{~kg}$ (BMI $15.0 \mathrm{~kg} / \mathrm{sqm}, 2$ percentile, $-2.16 \mathrm{SD}$ ) at the end of the first in hospital week. Up to the 29 treatment day the bodyweight increased by $8.5 \mathrm{~kg}$ (at least $4.9 \mathrm{~kg}$ additional body water). Propranolol treatment induce a loss of bodyweight of $2 \mathrm{~kg}$ within 48 hours and further $2 \mathrm{~kg}$ at day 36 (3.5 kg loss of total body water). The calculation of total body fat $(+6.1 \mathrm{~kg})$ and lean body mass $(-3.7 \mathrm{~kg})$ are not plausible and may be related to the automatic fat correction of the software that should eliminate unwanted water fluctuations as declared by Data Input on their company homepage.

However the most impressive effect of refeeding is measured by 24 hours heart rate variability analysis: Refeeding induces an increase of mean heart rate of $35 \%$ and a dramatic decrease of heart rate variability indicated by SDNN (-70\%) and rMSSD (- $81 \%)$. The current pathophysiological interpretation is a shift of the autonomic nervous system from an extraordinary parasympathetic activation to sympathetic activation. This "collapse of the vagus" is shown by HRV monitoring and have to be overlooked using the current recommendations to monitor the refeeding syndrome based upon laboratory values (NT-Pro-BNP is not recommended) and standard ECG. We observe in part very long QT intervals and QT dispersions in the Holter ECG but not in the standard ECG. All these parameters indicate a high risk of sudden cardiac death and clearly show the

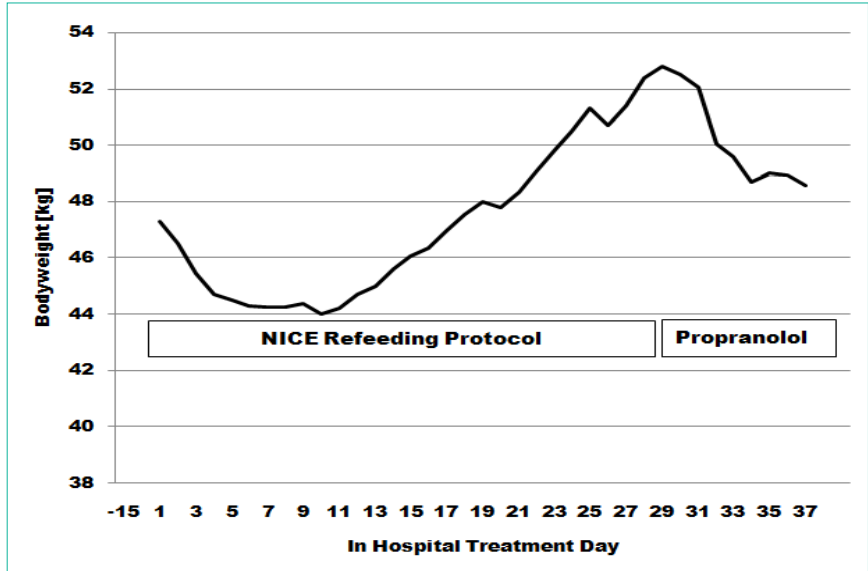

Figure 1: Daily measured bodyweight during refeeding of a girl with anorexia nervosa.
Citation: Buchhorn R, Bauman C and Willaschek C. Propranolol is Highly Effective to Treat Refeeding Oedema in a Girl with Anorexia Nervosa - A Case Report. Ann Nutr Disord \& Ther. 2017; 4(1): 1039. 
Table 1: Clinical data, bio impedance analysis, clinical chemistry and heart rate variability analysis during the course of refeeding.

\begin{tabular}{|c|c|c|c|c|c|c|}
\hline Day & Reference & -15 & 0 & 29 & 31 & 36 \\
\hline Bodyweight [kg] & & 47.8 & 47.3 & 52.5 & 50.5 & 48.6 \\
\hline Caloric Intake [kcal] & 2200 & 2500 & 200 & 3500 & 2500 & 2500 \\
\hline Body fat [kg] & 13.8-25.6 & & -2.7 & 1.9 & 2.8 & 12.6 \\
\hline Total body fat [\%] & $5.7-14.3$ & & -1.2 & 1 & 1.4 & 6.1 \\
\hline Total Body water [l] & $23.5-32.1$ & & 27.9 & 31.8 & 30.5 & 28.3 \\
\hline Lean body mass [kg] & $33.7-44.9$ & & 46.2 & 51.5 & 49.1 & 42.5 \\
\hline Phase angle [degree] & $5.3-6.3$ & & 2.8 & 2.4 & 2.7 & 3 \\
\hline Hematocrit [\%] & $34-44$ & 41.5 & 40.4 & 33.7 & 34 & 42 \\
\hline Phosphat [mmol/l] & $0.8-1.5$ & & 1.2 & 1.4 & 1.5 & \\
\hline $\mathrm{K}^{+}[\mathrm{mmol} / \mathrm{l}]$ & $3.5-5.1$ & 3.94 & 4.23 & 4.99 & 5.01 & \\
\hline $\mathrm{Na}^{+}[\mathrm{mmol} / \mathrm{l}]$ & $135-150$ & 144 & 143 & 143 & 144 & \\
\hline $\mathrm{Ca}^{++}[\mathrm{mmol} / \mathrm{l}]$ & $2.15-2.75$ & 2.46 & 2.45 & 2.3 & 2.3 & \\
\hline $\mathrm{Mg}^{++}[\mathrm{mmol} / \mathrm{l}]$ & $0.66-0.91$ & & 1 & 0.9 & 0.8 & \\
\hline NT - BNP [pg/ml] & $0-145$ & & & 868 & 372 & 393 \\
\hline Proteine $[\mathrm{g} / \mathrm{dl}]$ & 6.1-8.3 & 7.1 & 7 & 6.2 & & 6.9 \\
\hline Heartrate $[\mathrm{bpm}]$ & $73-90$ & & 68 & 92 & 78 & 76 \\
\hline SDNN [ms] & $116-165$ & & 367.4 & 108.5 & 123.1 & 119.6 \\
\hline rMSSD [ms] & $35-70$ & & 174.4 & 33.8 & 43.3 & 46.3 \\
\hline QTcmax [ms] & $<440 \mathrm{~ms}$ & & 511 & 496 & 445 & 478 \\
\hline QTcdispersion [ms] & $<65 \mathrm{~ms}$ & & 138 & 156 & 77 & 106 \\
\hline
\end{tabular}

beneficial effect of propranolol on heart rate, QTc intervals, QTc dispersion and NT-Pro-BNP (Table 1).

With respect to the effect of propranolol treatment on refeeding oedema we have again the same enthusiastic discussions like 20 years ago if we treat the first children with severe heart failure due to congenital heart disease with propranolol. Obviously we now initiate the next step to improve pharmacological treatment in pediatrics based upon this controversial pathophysiological model by using propranolol for following indications:

1. 1995 we treat the first child with effusions after the Fontan procedure.
2. 1996 we treat the first infant with severe "heart failure" due to a single ventricle.

3. 2003 we treat the first infant with "heart failure" due to liver hemangioma.

4. 2017 we treat the first child with refeeding oedema.

Today we realize - most of all after intensive investigations according propranolol treatment of hemangioma in infancy - which the therapeutic success of propranolol in these four indications with normal ventricular function predominantly based upon its vascular effects and not on its effect on so called "heart failure". In contrast to the beta blocker therapy of heart failure we observe an immediate clinical improvement within the first treatment week probably based upon the effect on the capillary filtration pressure. The effect of beta blockers on the heart most of all due to cardiac remodeling needs longer time for clinical improvement if the drug have to be up titrated over weeks in order to prevent unwanted side effects.

Our pathophysiological model and the new therapeutic approach with propranolol may have important implication for children with refeeding edema who have a significant mortality.

Funding: We thank the aBlaschek foundation ${ }^{\circ}$ who supports us by covering the publication fees for this research project. The funders had no role in study design, data collection and analysis, decision to publish, or preparation of the manuscript.

\section{References}

1. Ehrlich S, Querfeld U, Pfeiffer E. Refeeding oedema: an important complication in the treatment of anorexia nervosa. Eur.Child Adolesc. Psychiatry. 2006; 15: 241-243.

2. Rytter MJ, Babirekere-Iriso E, Namusoke H, Christensen VB, Michaelsen KF, Ritz C, et al. Risk factors for death in children during inpatient treatment of severe acute malnutrition: a prospective cohort study. Am.J.Clin.Nutr. 2016.

3. Jauregui-Garrido B, Jauregui-Lobera I. Sudden death in eating disorders. Vasc. Health Risk Manag. 2012; 8: 91-98.

4. Dippacher S, Willaschek C, Buchhorn R. Different nutritional states and autonomic imbalance in childhood. Eur.J.Clin.Nutr. 2014; 68: 1271-1273.

5. Buchhorn R, Motz R. Hepatic encephalopathy and ascites. Lancet. 1998; 351: 448-449.
Ann Nutr Disord \& Ther - Volume 4 Issue 1 - 2017 ISSN : 2381-8891 | www.austinpublishinggroup.com Buchhorn et al. () All rights are reserved
Citation: Buchhorn R, Bauman C and Willaschek C. Propranolol is Highly Effective to Treat Refeeding Oedema in a Girl with Anorexia Nervosa - A Case Report. Ann Nutr Disord \& Ther. 2017; 4(1): 1039. 